December 27, 2017

\title{
On the Flattening of Negative Curvature via T-Duality with a Non-Constant $B$-field
}

\author{
Axel Krause ${ }^{1}$ \\ Humboldt-Universität, Institut für Physik, D-10115 Berlin, Germany
}

\begin{abstract}
In an earlier paper, Alvarez, Alvarez-Gaumé, Barbón and Lozano pointed out, that the only way to "flatten" negative curvature by means of a T-duality is by introducing an appropriate, non-constant NS-NS 2-form $B$. In this paper, we are investigating this further and ask, whether it is possible to T-dualize $\operatorname{AdS}_{d}$ space to flat space with some suitably chosen $B$. To answer this question, we derive the relation between the original curvature tensor and the one of the T-dualized metric involving the $B$-field. It turns out that there is one component which is independent of $B$. By inspection of this component we show that it is not possible to dualize $\mathrm{AdS}_{d}$ to flat space irrespective of the choice of $B$. Finally, we examine the extension of AdS to an $\mathrm{AdS}_{5} \times \mathrm{S}^{5}$ geometry and propose a chain of S- and T-dualities together with an $S L(2, \mathbb{Z})$ coordinate transformation, leading to a dual D9-brane geometry.
\end{abstract}

PACS: $11.25 .-\mathrm{w}$

Keywords: T-Duality, NS-NS B-Field

\footnotetext{
${ }^{1}$ E-mail: krause@physics.umd.edu; now at Department of Physics, University of Maryland, College Park, MD 20742, USA
} 
By now the AdS-CFT conjecture [1, 2, 3, has passed an enormous amount of tests (see [4] for a review). Most of them explored the large $N$ duality between the boundary-CFT and the supergravity on $\mathrm{AdS}_{5}$. The common radius of the $\mathrm{S}^{5}$ and the length-scale of the $\mathrm{AdS}_{5}, R$, is given by $R^{4}=\left(\alpha^{\prime}\right)^{2} \lambda$, with $\lambda=g_{Y M}^{2} N$ representing the 't Hooft coupling. In general supergravity as the low-energy limit of string-theory is trustworthy only at large scales, i.e. small curvatures. Thus tests of the duality probing the supergravity regime explore the $\lambda \rightarrow \infty$ parameter region. This predicts how the CFT at large $N$ behaves in the extreme non-perturbative regime.

The interesting parameter regime, interpolating between the perturbative $\lambda \rightarrow 0$ and the extreme non-perturbative $\lambda \rightarrow \infty$ regime, demands that we keep $\lambda$ finite. Since the closed string coupling constant $g_{s}$ and the Yang-Mills coupling constant are related via $g_{Y M}^{2}=4 \pi g_{s}$, the large $N$ limit with $\lambda$ finite, requires $g_{s} \rightarrow 0$. Thus via the AdS-CFT conjecture, we are able to extract the full quantum information about the CFT in the large $N$ limit by calculating simply IIB string tree-diagrams. Unfortunately, this wonderful perspective is obstructed by the fact, that IIB string-theory in the RNS-formulation on an $\mathrm{AdS}_{5} \times \mathrm{S}^{5}$ background with $N$ units of RR 5 -form flux through the $\mathrm{S}^{5}$ is still obscure (see [5] for an approach). On the other hand there are proposals for a GS-formulation 6] which is non-linear and therefore its quantization and computation of string scattering amplitudes seems to be difficult.

Since string-theory on an $\mathrm{AdS}_{5} \times \mathrm{S}^{5}$ background is not readily available, one may seek resort to a dual description of IIB string-theory on a, hopefully, easier background. As a first step into this direction, we want to analyze in this paper, whether pure $\mathrm{AdS}_{d}$ space can be dualized to flat Minkowski space. This would have important consequences for the cosmological constant in string-theory brane-worlds based on a bulk $\mathrm{AdS}_{d}$ space [7. The concrete motivation for this problem stems from the observation, made in [8], that the only way to "flatten" negative curvature under T-duality is by introducing an appropriate torsion, generated by $B_{\mu \nu}$, in the initial space-time. This can be seen from the following formula ${ }^{2}$, relating the dual curvature scalar $\tilde{R}$ to the initial curvature scalar 8

$$
\tilde{R}=R+4 \triangle \ln k+\frac{1}{k^{2}} H_{\iota \alpha \beta} H^{\iota \alpha \beta}-\frac{k^{2}}{4} F_{\alpha \beta} F^{\alpha \beta} .
$$

Here $A_{\alpha}=k_{\alpha} / k^{2}, k_{\alpha}=g_{\iota \alpha}$ with the associated field-strength $F_{\alpha \beta}=\partial_{\alpha} A_{\beta}-\partial_{\beta} A_{\alpha}$. Furthermore, $\triangle \ln k=\frac{1}{\sqrt{-g}} \partial_{\alpha}\left(\sqrt{-g} g^{\alpha \beta} \partial_{\beta} \ln k\right)$ denotes the d'Alembertian with respect to the initial metric. As usual, the torsion 3-form is the field strength ${ }^{3} H=d B$ of the NSNS 2-form $B_{\mu \nu}$. The Killing vector, corresponding to the assumed translational isometry exhibited by the initial space-time, is given by $k_{\mu}$ and its norm defined by $k=\sqrt{g_{\mu \nu} k^{\mu} k^{\nu}}$. One could now try to solve (II) for $R=-\Lambda$ and $\tilde{R}=0$, with $\Lambda$ a positive constant. But

\footnotetext{
${ }^{2}$ The index $\iota$ represents the isometry direction, whereas $\alpha, \beta, \ldots$ label the remaining directions.
}

${ }^{3}$ Our conventions are:

$$
B=\frac{1}{2 !} B_{\mu \nu} d x^{\mu} \wedge d x^{\nu}, \quad H=\frac{1}{3} H_{\mu \nu \rho} d x^{\mu} \wedge d x^{\nu} \wedge d x^{\rho} .
$$


this would include any solution of the vacuum Einstein equations. To decide whether we arrive at flat space after T-dualization, we have to regard the Riemann curvature tensor.

Let us start quite generally by assuming some coordinate representation of a $d$ dimensional manifold, given by ${ }^{4} x^{\mu}=\left(x^{0}, x^{1}, \ldots, x^{d-2}, x^{d-1}\right)=\left(x^{\alpha}, x^{\iota}\right) ; \alpha=0, \ldots, d-2$. Furthermore, the initial metric is supposed to be of the form

$$
d s^{2}=g_{\alpha \beta} d x^{\alpha} d x^{\beta}+g_{\iota \iota} d x^{\iota} d x^{\iota}
$$

From the Buscher rules for T-duality

$$
\tilde{g}_{\iota \iota}=\frac{1}{g_{\iota \iota}}, \quad \tilde{g}_{\iota \alpha}=\frac{B_{\iota \alpha}}{g_{\iota \iota}}, \quad \tilde{g}_{\alpha \beta}=g_{\alpha \beta}-\frac{\left(g_{\iota \alpha} g_{\iota \beta}-B_{\iota \alpha} B_{\iota \beta}\right)}{g_{\iota \iota}},
$$

we observe, that our choice of $g_{\iota \alpha}=0$ cannot be compensated by an appropriate choice of some real-valued $B_{\iota \alpha}$ (only an imaginary would suffice). The reason why we set nevertheless $g_{\iota \alpha}=0$, from the outset, is the following. According to (11), a non-vanishing $g_{\iota \alpha}=k_{\alpha}$ tends to make the curvature scalar of the dual metric more negative. Since we start with a negatively curved $\mathrm{AdS}_{d}$ and aim to bring it to flat space, a $k_{\alpha} \neq 0$ would obstruct our intention.

We assume that the metric $g_{\mu \nu}\left(x^{\alpha}\right)$ and the NS-NS 2-form $B_{\mu \nu}\left(x^{\alpha}\right)$ do not depend on $x^{\iota}$. These conditions are exactly those, which are required in order to leave the $\sigma$-model action

$$
S=\frac{1}{2 \pi} \int d^{2} z\left(g_{\mu \nu}+B_{\mu \nu}\right) \partial X^{\mu} \bar{\partial} X^{\nu}
$$

invariant under infinitesimal shifts $x^{\mu} \rightarrow x^{\mu}+\epsilon k^{\mu}$. The Killing vector associated with the resulting abelian translational isometry, is $k^{\mu} \partial_{\mu}=\partial / \partial x^{\iota}$, with $k^{\mu}=\left(k^{\alpha}, k^{\iota}\right)=(0, \ldots, 1)$. With the resulting norm $k=\sqrt{g_{\iota \iota}}$ of the Killing vector, a more convenient expression for the metric (2) in view of our later application, is given in terms of an anholonomic vielbein co-base by

$$
d s^{2}=\eta_{a b} e^{a} e^{b}+e^{i} e^{i}=\eta_{m n} e^{m} e^{n},
$$

where the chosen anholonomic vielbein $e^{l}=\left(e^{a}, e^{i}\right)$ reads

$$
e^{a}=e^{a}{ }_{\alpha}\left(x^{\beta}\right) d x^{\alpha}, \quad \quad e^{i}=e^{i}{ }_{\iota} d x^{\iota}=\delta_{\iota}^{i} k\left(x^{\alpha}\right) d x^{\iota},
$$

such that its components $e_{\lambda}^{l}$ are

$$
\begin{array}{ll}
e^{a}{ }_{\alpha}=e^{a}{ }_{\alpha}\left(x^{\beta}\right), & e^{i}{ }_{\alpha}=0 \\
e^{a}{ }_{\iota}=0, & e^{i}{ }_{\iota}=\delta_{\iota}^{i} k .
\end{array}
$$

Later, we will also need the inverted vielbein of the isometry direction $e_{i}^{\iota}=\delta_{i}^{\iota} / k$.

The above mentioned $\sigma$-model action is invariant 9] to first order in $\epsilon$ under the translational isometry $\delta_{\epsilon} x^{\mu}=\epsilon k^{\mu}$, if $k^{\mu}$ satisfies the Killing equation $\left(\mathcal{L}_{k} g\right)_{\mu \nu}=k^{\lambda} g_{\mu \nu, \lambda}+$ $k_{, \mu}^{\lambda} g_{\lambda \nu}+k_{, \nu}^{\lambda} g_{\lambda \mu}=\partial g_{\mu \nu} / \partial x^{\iota}=0$ and the torsion obeys $\mathcal{L}_{k} H=0$. This implies $\mathcal{L}_{k} B=d w$

\footnotetext{
${ }^{4}$ Indices $\lambda, \mu, \nu, \pi$ run from $0, \ldots, d-1$, whereas indices $\alpha, \beta, \gamma, \delta, \epsilon$ run over $0, \ldots, d-2$.
} 
for some 1 -form $w$. Here $\mathcal{L}_{k}$ and $d$ denote the space-time Lie- and exterior derivative. Locally, this is solved by $w=i_{k} B-v$ with $d v=-i_{k} H$ for some 1-form $v$. Under $i_{k}$ we understand the interior product. From now on, we will choose the gauge $w=0$, which leads us, together with the identity $\left(i_{k} B\right)_{\mu}=k^{\nu} B_{\nu \mu}=B_{\iota \mu}$, to

$$
v_{\alpha}=B_{\iota \alpha}
$$

The T-dual metric $\tilde{g}_{\mu \nu}$ reduces in the case of $k^{\alpha}=0$ to

$$
\tilde{g}_{\iota \iota}=\frac{1}{k^{2}}, \quad \tilde{g}_{\alpha \iota}=\frac{v_{\alpha}}{k^{2}}, \quad \tilde{g}_{\alpha \beta}=g_{\alpha \beta}+\frac{v_{\alpha} v_{\beta}}{k^{2}} .
$$

Thus the dual line-element can be written as

$$
d \tilde{s}^{2}=\tilde{g}_{\alpha \beta} d x^{\alpha} d x^{\beta}+2 \tilde{g}_{\alpha \iota} d x^{\alpha} d x^{\iota}+\tilde{g}_{\iota \iota} d x^{\iota} d x^{\iota}=\eta_{a b} \tilde{e}^{a} \tilde{e}^{b}+\tilde{e}^{i} \tilde{e}^{i}=\eta_{m n} \tilde{e}^{m} \tilde{e}^{n} .
$$

Here the T-dual anholonomic vielbein co-base $\tilde{e}^{l}=\left(\tilde{e}^{a}, \tilde{e}^{i}\right)$ is given by

$$
\tilde{e}^{a}=e^{a}=e^{a}{ }_{\alpha} d x^{\alpha}, \quad \quad \tilde{e}^{i}=\tilde{e}_{\mu}^{i} d x^{\mu}=\frac{\delta_{\iota}^{i}}{k} d x^{\iota}+\frac{v_{\alpha}}{k} d x^{\alpha}
$$

from which we can read off the dual vielbein components $\tilde{e}_{\lambda}^{l}$

$$
\begin{aligned}
\tilde{e}^{a}{ }_{\alpha} & =e^{a}{ }_{\alpha}, & \tilde{e}^{i}{ }_{\alpha} & =\frac{v_{\alpha}}{k} \\
\tilde{e}^{a}{ }_{\iota} & =0, & \tilde{e}^{i}{ }_{\iota} & =\frac{\delta_{\iota}^{i}}{k} .
\end{aligned}
$$

and their inverses $\tilde{e}_{l}^{\lambda}$

$$
\begin{array}{ll}
\tilde{e}_{a}^{\alpha}=e_{a}^{\alpha}, & \tilde{e}_{i}^{\alpha}=0 \\
\tilde{e}_{a}^{\iota}=-e_{a}^{\alpha} v_{\alpha}, & \tilde{e}_{i}^{\iota}=\delta_{i}^{\iota} k .
\end{array}
$$

A useful formula consists of the inverted relation $\delta_{\iota}^{i} d x^{\iota}=k \tilde{e}^{i}-e_{a}{ }^{\alpha} v_{\alpha} e^{a}$. From $\tilde{g}^{\mu \nu}=$ $\tilde{e}_{m}{ }^{\mu} \tilde{e}_{n}{ }^{\nu} \eta^{m n}$ we derive the inverse metric to be

$$
\tilde{g}^{\alpha \beta}=g^{\alpha \beta}, \quad \tilde{g}^{\alpha \iota}=-g^{\alpha \beta} v_{\beta}, \quad \tilde{g}^{\iota \iota}=g^{\alpha \beta} v_{\alpha} v_{\beta}+k^{2} .
$$

The strategy of the calculation of the dual Riemann-tensor will now be as follows. With the aid of Cartan's structure equations, we determine the curvature tensor and its T-dual in the $e^{l}=\left\{e^{a}, e^{i}\right\}$, resp. T-dual $\tilde{e}^{l}=\left\{\tilde{e}^{a}, \tilde{e}^{i}\right\}$ co-base. In order to compare both of them, it is further necessary to switch to the equivalent expressions in the common holonomic co-base $d x^{\mu}=\left(d x^{\alpha}, d x^{\iota}\right)$ with the help of the aforementioned vielbeins.

Therefore, let us begin with (3) and avail ourselves of Cartan's first structure equation for the torsion-less case, $d e^{m}+\omega^{m}{ }_{n} \wedge e^{n}=0$, to determine the connection 1-form $\omega$

$$
\omega_{b}^{a}=-\partial_{[\gamma} e^{a}{ }_{\alpha]} e_{[c}^{\gamma} e_{b]}^{\alpha} e^{c}, \quad \omega_{a}^{i}=e_{a}^{\alpha} \partial_{\alpha} \ln k \cdot e^{i}, \quad \omega_{i}^{i}=0
$$


Cartan's second structure equation, $d \omega^{m}{ }_{n}+\omega^{m}{ }_{l} \wedge \omega_{n}^{l}=R_{n}^{m}$, together with the expression for the curvature 2-form in the initial anholonomic co-base, $R_{m}^{l}=\frac{1}{2} R_{m n p}^{l} e^{n} \wedge e^{p}=$ $\frac{1}{2} R_{\text {mab }}^{l} e^{a} \wedge e^{b}+R_{\text {mia }}^{l} e^{i} \wedge e^{a}$, allow us to extract after some algebra the initial curvature tensor ${ }^{5}$ as

$$
\begin{aligned}
R_{a b c d} & =e_{[c}^{\gamma} e_{d]}^{\delta}\left(e_{b}^{\beta} \partial_{\beta} \partial_{\gamma} e_{a \delta}-\partial_{[\delta} e_{|a| \beta]} \cdot \partial_{\gamma} e_{b}^{\beta}\right)+e_{b}^{\beta} e_{[c}^{\gamma} \partial_{|\beta|} e_{d]}^{\alpha} \cdot \partial_{[\gamma} e_{|a| \alpha]} \\
R_{i a b c} & =R_{a b i c}=0 \\
R_{\text {iaib }} & =-e_{(a}^{\alpha} \partial_{|\alpha|} e_{b)}{ }^{\beta} \cdot \partial_{\beta} \ln k-e_{a}^{\alpha} e_{b}^{\beta}\left(\partial_{\alpha} \partial_{\beta} \ln k+\partial_{\alpha} \ln k \cdot \partial_{\beta} \ln k\right),
\end{aligned}
$$

whereas all other components are zero. By multiplying with the vielbein $R_{\lambda \mu \nu \pi}=e_{\lambda}^{l} e_{\mu}^{m}$ $e^{n}{ }_{\nu} e_{\pi}^{p} R_{l m n p}$, we subsequently arrive at the coordinate base expressions

$$
\begin{aligned}
R_{\alpha \beta \gamma \delta} & =\delta_{[\gamma}^{\varepsilon} e_{\delta]}^{b}\left(e_{a \alpha} \partial_{\beta} e_{b}^{\zeta} \cdot \partial_{[\varepsilon} e_{\zeta]}^{a}+\frac{1}{2} e_{a}^{\zeta} \partial_{\zeta} e_{b \alpha} \cdot \partial_{\varepsilon} e_{\beta}^{a}\right)+e_{\alpha}^{a} \partial_{\beta} \partial_{[\gamma} e_{|a| \delta]}+\frac{1}{2} \partial_{[\gamma} e_{|\alpha|}^{a} \cdot \partial_{\delta]} e_{a \beta} \\
R_{\iota \alpha \beta \gamma} & =R_{\alpha \beta \iota \gamma}=0 \\
R_{\iota \alpha \iota \beta} & =-k^{2}\left(e^{a}{ }_{(\alpha} \partial_{\beta} e_{a} e^{\varepsilon} \cdot \partial_{\varepsilon} \ln k+\partial_{\alpha} \partial_{\beta} \ln k+\partial_{\alpha} \ln k \cdot \partial_{\beta} \ln k\right),
\end{aligned}
$$

and all other components zero.

Analogously, application of $d \tilde{e}^{l}+\tilde{\omega}_{m}^{l} \wedge \tilde{e}^{m}$ yields

$$
\tilde{\omega}_{b}^{a}=\omega_{b}^{a}, \quad \tilde{\omega}_{a}^{i}=-e_{b}{ }^{\beta} e_{a}^{\alpha} \frac{d v_{\beta \alpha}}{k} e^{b}-e_{a}^{\alpha} \partial_{\alpha} \ln k \tilde{e}^{i}, \quad \tilde{\omega}_{i}^{i}=0,
$$

where $d v_{\alpha \beta} \equiv \partial_{[\alpha} v_{\beta]}$ denotes the exterior derivative of $v$. From the relation $d v=-i_{k} H$, it is possible to substitute $d v_{\alpha \beta}$ in the subsequent formulae by the torsion 3-form through $d v_{\alpha \beta}=-H_{\iota \alpha \beta}$. Again, from Cartan's second structure equation, $d \tilde{\omega}^{m}{ }_{n}+\tilde{\omega}^{m}{ }_{l} \wedge \tilde{\omega}_{n}^{l}=\tilde{R}_{n}^{m}$, and the dual curvature 2-form $\tilde{R}_{m}^{l}=\frac{1}{2} \tilde{R}_{m n p}^{l} \tilde{e}^{n} \wedge \tilde{e}^{p}=\frac{1}{2} \tilde{R}_{m a b}^{l} e^{a} \wedge e^{b}+\tilde{R}_{m i a}^{l} \tilde{e}^{i} \wedge e^{a}$, we get the following non-coordinate frame expressions

$$
\begin{aligned}
\tilde{R}_{a b c d} & =R_{a b c d}-\frac{2}{k^{2}} e_{a}^{\alpha} e_{b}^{\beta} e_{[c}^{\gamma} e_{d]}^{\delta} d v_{\alpha \gamma} d v_{\beta \delta} \\
\tilde{R}_{i a b c} & =\tilde{R}_{b c i a}=-\frac{2}{k} e_{a}^{\alpha} e_{[b}^{\beta} e_{c]}^{\gamma} d v_{\beta \alpha} \cdot \partial_{\gamma} \ln k \\
\tilde{R}_{i a i b} & =e_{(a}^{\alpha} \partial_{|\alpha|} e_{b)}{ }^{\beta} \cdot \partial_{\beta} \ln k+e_{a}^{\alpha} e_{b}^{\beta}\left(\partial_{\alpha} \partial_{\beta} \ln k-\partial_{\alpha} \ln k \cdot \partial_{\beta} \ln k\right) .
\end{aligned}
$$

Switching to the coordinate base with the help of $\tilde{R}_{\lambda \mu \nu \pi}=\tilde{e}_{\lambda}^{l} \tilde{e}^{m}{ }_{\mu} \tilde{e}^{n}{ }_{\nu} \tilde{e}^{p}{ }_{\pi} \tilde{R}_{l m n p}$, one finally arrives, after some tedious algebra, at the desired expressions, which relate the T-dual

\footnotetext{
${ }^{5}$ We use the following anti- and symmetrization convention:
}

$$
A_{(a b)}=\frac{1}{2}\left(A_{a b}+A_{b a}\right) \quad A_{[a b]}=\frac{1}{2}\left(A_{a b}-A_{b a}\right)
$$


curvature tensor to its counterpart for the initial metric

$$
\begin{gathered}
\tilde{R}_{\alpha \beta \gamma \delta}=R_{\alpha \beta \gamma \delta}+\frac{2}{k^{2}}\left(d v_{\alpha[\delta} d v_{|\beta| \gamma]}-d v_{\alpha \gamma} \cdot v_{[\beta} \partial_{\delta]} \ln k+d v_{\beta \gamma} \cdot v_{[\alpha} \partial_{\delta]} \ln k+d v_{\alpha \delta} \cdot v_{[\beta} \partial_{\gamma]} \ln k\right. \\
-d v_{\beta \delta} \cdot v_{[\alpha} \partial_{\gamma]} \ln k+v_{[\alpha} e_{\beta]}^{b} v_{[\gamma} \partial_{\delta]} e_{b}^{\varepsilon} \cdot \partial_{\varepsilon} \ln k+v_{[\gamma} e_{\delta]}^{b} v_{[\alpha} \partial_{\beta]} e_{b}^{\varepsilon} \cdot \partial_{\varepsilon} \ln k \\
\left.+v_{\gamma} v_{[\alpha} \partial_{\beta]} \partial_{\delta} \ln k-v_{\delta} v_{[\alpha} \partial_{\beta]} \partial_{\gamma} \ln k-2 v_{[\alpha} \partial_{\beta]} \ln k \cdot v_{[\gamma} \partial_{\delta]} \ln k\right) \\
\tilde{R}_{\iota \alpha \beta \gamma}=\frac{2}{k^{2}}\left(d v_{\alpha[\beta} \cdot \partial_{\gamma]} \ln k+\frac{1}{2}\left(e_{\alpha}^{a} v_{[\beta} \partial_{\gamma]} e_{a}^{\delta}+v_{[\beta} e_{\gamma]}^{a} \partial_{\alpha} e_{a}^{\delta}\right) \cdot \partial_{\delta} \ln k+v_{[\beta} \partial_{\gamma]} \partial_{\alpha} \ln k\right. \\
\left.\quad-v_{[\beta} \partial_{\gamma]} \ln k \cdot \partial_{\alpha} \ln k\right) \\
\tilde{R}_{\iota \alpha \iota \beta}=-\frac{1}{k^{2}}\left(\frac{R_{\iota \alpha \iota \beta}}{k^{2}}+2 \partial_{\alpha} \ln k \cdot \partial_{\beta} \ln k\right) .
\end{gathered}
$$

As an application of these formulae, we now want to examine, whether d-dimensional $\mathrm{AdS}_{d}$ space allows a T-dualization to flat space under the inclusion of some suitably chosen $B_{\mu \nu}$. Because both spaces, regarded as string backgrounds, leave all supersymmetries intact this is a reasonable question to ask. We choose the following coordinate representation for $\mathrm{AdS}_{d}$

$$
d s^{2}=\frac{r^{2}}{L^{2}}\left(-d t^{2}+\sum_{j=1}^{d-2} d y^{j} d y^{j}\right)+L^{2} \frac{d r^{2}}{r^{2}}
$$

with $L$ being the AdS-radius. It exhibits a negative, constant curvature scalar $R=$ $-d(d-1) / L^{2}$. Let us first choose for the isometry direction one of the $y^{j}$ coordinates, e.g. $y^{d-2}$. We will see below how the results for this case extrapolate to the case of a different isometry direction. In our above convention, the adapted coordinates are $x^{0}=t, x^{1}=y^{1}, \ldots, x^{d-3}=y^{d-3}, x^{d-2}=r, x^{d-1} \equiv x^{\iota}=y^{d-2}$. The curvature tensor of the negatively curved, maximally symmetric $\mathrm{AdS}_{d}$ is given by $R_{\lambda \mu \nu \pi}=-\frac{1}{L^{2}}\left(g_{\lambda \nu} g_{\mu \pi}-g_{\lambda \pi} g_{\mu \nu}\right)$, which reduces in our parameterization to ${ }^{6}$

$$
R_{\mu \nu \mu \nu}=-\frac{r^{4}}{L^{6}} \eta_{\mu \mu} \eta_{\nu \nu}, \quad R_{\mu r \mu r}=-\frac{1}{L^{2}} \eta_{\mu \mu}, \quad \mu, \nu \neq r
$$

and all other components vanishing. In particular, this gives $R_{\iota \alpha \iota \beta}=-\frac{r^{4}}{L^{6}} \eta_{\alpha \beta}+\left(\frac{r^{4}}{L^{6}}-\right.$ $\left.\frac{1}{L^{2}}\right) \delta_{\alpha}^{r} \delta_{\beta}^{r}$. Now, we had found one component, (13), of the dual curvature-tensor, which was independent of $v$, resp. $B_{\mu \nu}$ and hence allows answering the above posed question. Plugging in the actual norm of the Killing vector $k=r / L$ for the above $\mathrm{AdS}_{d}$ metric, we obtain for the right-hand side of (13) the expression $\frac{1}{L^{2}} \eta_{\alpha \beta}-\left(\frac{1}{L^{2}}+\frac{L^{2}}{r^{4}}\right) \delta_{\alpha}^{r} \delta_{\beta}^{r}$. Obviously, this does not fulfill the requirement $\tilde{R}_{\iota \alpha \iota \beta}=0$ for flat space and is not even asymptotically flat.

\footnotetext{
${ }^{6}$ We choose the Minkowski metric to be $\eta=(-,+, \ldots,+)$.
} 
This can be extrapolated to any other isometry direction of $\mathrm{AdS}_{d}$ by noting that another isometry direction different from the above choice of $y^{d-2}$ can be related to this one by a general coordiante transformation $x \rightarrow x^{\prime}$. However, the tensor transformation property $\tilde{R}_{\iota^{\prime} \alpha^{\prime} \iota^{\prime} \beta^{\prime}}^{\prime}\left(x^{\prime}\right)=\left(A_{\iota^{\prime}}^{\iota}\right)^{2} A_{\alpha^{\prime}}^{\alpha} A_{\beta^{\prime}}^{\beta} \tilde{R}_{\iota \alpha \iota \beta}(x)$, where $A_{\nu}^{\mu}=\partial x^{\mu} / \partial x^{\prime \nu}$, then guarantees that this term is still non-vanishing in contrast to what one would need for a dual flat spacetime. Thus, we can conclude that flat space cannot be reached from pure $\mathrm{AdS}_{d}$ via T-duality under the inclusion of some appropriately chosen $B_{\mu \nu}$.

Eventually, we turn briefly to the important $\mathrm{AdS}_{5} \times \mathrm{S}^{5}$ extension of AdS. Here it seems possible to relate the IIB D9-brane geometry, which describes $\mathrm{D}=10$ space itself, to the $\mathrm{AdS}_{5} \times \mathrm{S}^{5}$ geometry. In order to do so, one has to start with a six-fold T-duality, transforming the D9-brane to the D3-brane. Then, dualizing a IIB D3-brane to its own near-horizon geometry, one reaches $\mathrm{AdS}_{5} \times S^{5}$. This can be done following the work of 10. Having T-dualized the D9-brane, the D3-brane geometry in string-frame reads

$$
\begin{array}{rlrl}
d s^{2} & =\frac{1}{\sqrt{H_{6}}}\left(-d t^{2}+\sum_{i=1}^{3}\left(d x^{i}\right)^{2}\right)+\sqrt{H_{6}} \sum_{j=4}^{9}\left(d x^{j}\right)^{2} \\
e^{\phi} & =1, & C_{0123} & =\frac{1}{H_{6}}-1 \\
H_{6} & =1+\frac{Q_{D 3}}{\left(r_{6}\right)^{4}}, & Q_{D 3} & =4 \pi g_{s} N l_{s}^{4} .
\end{array}
$$

Performing a further T-duality over $x^{3}, x^{2}$ brings us to the IIB D1-brane. An S-duality transformation then yields the fundamental IIB string solution

$$
\begin{array}{rlrl}
d s^{2} & =\frac{1}{H_{8}}\left(-d t^{2}+\left(d x^{1}\right)^{2}\right)+\sum_{j=2}^{9}\left(d x^{j}\right)^{2}, \\
e^{\phi} & =\frac{1}{\sqrt{H_{8}}}, & B_{01} & =\frac{1}{H_{8}}-1, \\
H_{8} & =1+\frac{Q_{F 1}}{\left(r_{8}\right)^{6}}, & Q_{F 1} & =d_{1} g_{s}^{2} N l_{s}^{6},
\end{array}
$$

which is then T-dualized over $x_{1}$ to obtain the IIA gravitational wave solution

$$
\begin{gathered}
d s^{2}=\left(H_{8}-2\right) d t^{2}+2\left(H_{8}-1\right) d t d x^{1}+H_{8} d x^{1} d x^{1}+\sum_{j=2}^{9}\left(d x^{j}\right)^{2}, \\
e^{\phi}=1, \quad B_{\mu \nu}=0 .
\end{gathered}
$$

The crucial step to get rid of the constant in the harmonic $H_{8}$ function, consists of performing first an $S L(2, \mathbb{R})$ coordinate transformation on the coordinates $t, x^{1}$ and subsequently a T-duality transformation in the new $x^{\prime 1}$ direction. Provided that we choose the special $S L(2, \mathbb{R})$ coordinate transformation

$$
t^{\prime}=\frac{1}{2}\left(t+x^{1}\right), \quad x^{1}=2 x^{1}
$$


we are guaranteed that the transformation is globally well-defined on $\left(t, x^{1}\right)$ space, which has the topology of a cylinder due to the compactification of $x^{1}$. After T-duality in $x^{\prime 1}$ direction the result is a modified fundamental string without constant part in the harmonic function

$$
\begin{gathered}
d s^{2}=\frac{1}{\mathcal{H}_{8}}\left(-\left(d t^{\prime}\right)^{2}+\left(d x^{\prime 1}\right)^{2}\right)+\sum_{j=2}^{9}\left(d x^{j}\right)^{2} \\
e^{\phi}=\frac{1}{\sqrt{\mathcal{H}_{8}}}, \quad B_{01}=\frac{1}{\mathcal{H}_{8}}-2 \\
\mathcal{H}_{8}=\frac{Q_{F 1}}{\left(r_{8}\right)^{6}}
\end{gathered}
$$

It is important to note that the last T-duality has again been along a space-like direction. Therefore we are secured to stay in the Type II string-theory framework instead of changing to Type $\mathrm{II}^{*}$ [1].

Now, proceeding in the inverse manner, a second S-duality promotes us to a modified D1-brane. Ultimately, with two T-dualities in the $x^{2}, x^{3}$ directions we end up with a modified D3-brane solution without a constant part in its harmonic function. As is well-known, this gives the $\mathrm{AdS}_{5} \times \mathrm{S}^{5}$ geometry. Notice, however, that the D3-brane solution is only locally dual to the $\mathrm{AdS}_{5} \times \mathrm{S}^{5}$ solution. In order to perform the various T-dualities, the brane has to be wrapped on a torus with all worldvolume coordinates taken to be periodic. Therefore the coordinates of the final $\mathrm{AdS}_{5} \times \mathrm{S}^{5}$ geometry in addition have to be identified globally. As mentioned in [10] this has the effect that only half the Killing spinors of $\mathrm{AdS}_{5}$ remain. Thus, only locally we have a maximally supersymmetric $\mathrm{AdS}_{5} \times \mathrm{S}^{5}$ solution. Globally, the D3-brane as well as the dual solution break half the supersymmetry.

A word of caution is in order. The spacetime-filling D9-brane is supposed to describe flat ten-dimensional Minkowski spacetime [12. However, the D9-brane breaks half the supersymmetry, whereas flat space does not. The resolution to this puzzle comes from the well-known fact, that the open-string sector of $N$ D9-branes is only consistent, if we have $N=32$ of them and perform an orientifolding by the world-sheet parity $\Omega: \sigma \rightarrow \pi-\sigma$. This breaks half the supersymmetry and the resulting Type I SO(32) theory gives us in addition to the flat ten-dimensional Minkowski solution a spacetime-filling O9 orientifold fixed plane. It would be interesting to study the influence of dualities on the cosmological constant in string-theory more generally. In particular the strongly coupled heterotic string [13] whose non-perturbative open membrane boundary-boundary interactions lead

to a non-vanishing positive vacuum energy [14] (see also [15]) would be an interesting candidate to study.

\section{Acknowledgements}

Financial support by the DFG is gratefully acknowledged. 


\section{References}

[1] J. Maldacena, The large N Limit of Superconformal Field Theories and Supergravity, Adv. Theor. Math. Phys. 2 (1998) 231, hep-th/9711200,

[2] S.S. Gubser, I.R. Klebanov and A.M. Polyakov, Gauge Theory Correlators from Noncritical String Theory, Phys. Lett. B428 (98) 105, hep-th/9802109.

[3] E. Witten, Anti-de Sitter Space and Holography, Adv. Theor. Math. Phys. 2 (1998) 253, hep-th/9802150;

[4] O. Aharony, S.S. Gubser, J. Maldacena, H. Ooguri and Y. Oz, Large N Field Theories, String Theory and Gravity, hep-th/9905111;

[5] D. Polyakov, On the NSR Formulation of String Theory on $A d S_{5} \times S^{5}$, hep-th/9812044;

[6] R.R. Metsaev and A.A. Tseytlin, Supersymmetric D3-brane Action in AdS $S_{5} \times S^{5}$, Phys. Lett. B436 (1998) 281, hep-th/9806095 R. Kallosh and A.A. Tseytlin, Simplifying Superstring Action on $A d S_{5} \times S^{5}$, JHEP 9810 (1998) 016, hep-th/9808088; I. Pesando, A Kappa fixed Type IIB Superstring Action on $A d S_{5} \times S^{5}$, JHEP 9811 (1998) 002, hep-th/9808020 R. Kallosh, J. Rahmfeld and A. Rajaraman, Near Horizon Superspace, JHEP 9809 (1998) 002, hep-th/9805217; R. Kallosh and J. Rahmfeld, The GS String Action on $A d S_{5} \times S^{5}$, Phys. Lett. B443 (1998) 143 , hep-th/9808038;

[7] E. Verlinde, On $R G$ Flow and the Cosmological Constant, Class. Quant. Grav. 17 (2000) 1277, hep-th/9912058; C. Schmidhuber, ADS(5) and the $4 D$ Cosmological Constant, Nucl. Phys. B580 (2000) 140, hep-th/9912156, A. Krause, A Small Cosmological Constant, Grand Unification and Warped Geometry, hep-th/0006226; A Small Cosmological Constant and Backreaction of Non-Finetuned Parameters, JHEP 0309 (2003) 016, hep-th/0007233 Heterotic M-Theory, Warped Geometry and the Cosmological Constant Problem, Fortsch. Phys. 49 (2001) 163;

[8] E. Alvarez, L. Alvarez-Gaume, J.L.F. Barbon and Y. Lozano, Some Global Aspects of Duality in String Theory, Nucl. Phys. B415 (1994) 71, hep-th/9309039,

[9] M. Roček and E. Verlinde, Duality, Quotients and Currents, Nucl. Phys. B373 (1992) 630, hep-th/9110053

[10] H.J. Boonstra, B. Peeters and K. Skenderis, Duality and Asymptotic Geometries, Phys. Lett. B411 (1997) 59, hep-th/9706192;

[11] C. M. Hull, Timelike T-Duality, de Sitter Space, Large N Gauge Theories and Topological Field Theory, JHEP 9807 (1998) 021, hep-th/9806146. 
[12] E. Bergshoeff and M. de Roo, D-Branes and T-Duality, Phys. Lett. B380 (1996) 265, hep-th/9603123.

[13] P. Hořava and E. Witten, Heterotic and Type I String Dynamics from Eleven Dimensions, Nucl. Phys. B460 (1996) 506, hep-th/9510209; Eleven-Dimensional Supergravity on a Manifold with Boundary, Nucl. Phys. B475 (1996) 94, hep-th/9603142 E. Witten, Strong Coupling Expansion of Calabi-Yau Compactification, Nucl. Phys. B471 (1996) 135, hep-th/9602070; A. Lukas, B.A. Ovrut and D. Waldram, On the Four-Dimensional Effective Action of Strongly Coupled Heterotic String Theory, Nucl. Phys. B532 (1998) 43, hep-th/9710208, The Ten-Dimensional Effective Action of Strongly Coupled Heterotic String Theory, Nucl. Phys. B540 (1999) 230, hep-th/9801087; G. Curio and A. Krause, Four-Flux and Warped Heterotic M-Theory Compactifications, Nucl. Phys. B602 (2001) 172, hep-th/0012152,

[14] G. Moore, G. Peradze and N. Saulina, Instabilities in Heterotic M-Theory induced by Open Membrane Instantons, Nucl. Phys. B607 (2001) 117, hep-th/0012104 G. Curio and A. Krause, G-Fluxes and Non-Perturbative Stabilisation of Heterotic M-Theory, Nucl. Phys. B643 (2002) 131, hep-th/0108220.

[15] C. Bachas and B. Pioline, High-Energy Scattering on Distant Branes, JHEP 9912 (1999) 004, hep-th/9909171; A. Krause, Testing Stability of $M$-Theory on an $S^{1} / Z_{2}$ Orbifold, JHEP 0005 (2000) 046, hep-th/9909182, T. Dasgupta, M.R. Gaberdiel and M.B. Green, The Type I D-Instanton and its M-Theory Origin, JHEP 0008 (2000) 004, hep-th/0005211. 